\title{
Dynamic threshold computation in fault detection for discrete-time linear systems
}

\author{
Xinyang Liu ${ }^{1}$, Zhenhua Wang ${ }^{1}$, Ye Wang ${ }^{2}$, Yi Shen ${ }^{1}$ \\ 1. School of Astronautics, Harbin Institute of Technology, Harbin 150001, China \\ E-mail: zhenhua.wang@hit.edu.cn \\ 2. Institut de Robòtica i Informàtica Industrial, CSIC-UPC, Universitat Politècnica de Catalunya-BarcelonaTech (UPC), C/. Llorens i \\ Artigas 4-6, 08028, Barcelona, Spain
}

\begin{abstract}
This paper studies threshold computation in observer-based fault detection for discrete-time linear systems subject to unknown but bounded uncertainties. Based on two different assumptions on uncertainties, we propose two threshold computation methods for residual evaluation. First, we assume that the uncertainties are bounded in known intervals and propose a zonotope-based threshold computation method. Second, the uncertainties are assumed to be norm-bounded and then a threshold computation method using $L_{\infty}$ analysis is proposed. Numerical simulations are given to verify and compare the performance of the proposed methods.
\end{abstract}

Key Words: Fault detection, Threshold computation, Zonotopes, $L_{\infty}$ analysis.

\section{INTRODUCTION}

Fault diagnosis has attracted much attention in the past decades since it can be used to enhance the safety of control systems. In the existing results on fault diagnosis, modelbased methods have been most widely and intensively studied $[1,2,3]$. It is known that model-based fault diagnosis consists of two parts: residual generation and residual evaluation. The task of residual generation is to generate a residual signal that reflects fault occurrence and the function of residual evaluation is making decision so as to give fault diagnosis result. Although both parts are important in fault diagnosis, most of the existing methods of fault diagnosis focus on residual generation such as fault detection observer $[4,5,6]$ and parity space approach $[7,8]$. In contrast to residual generation, there are limited results on residual evaluation $[9,10,11,12]$.

In fault detection, residual evaluation is usually achieved by a comparison between the residual feature and a threshold. Therefore, threshold computation is significantly important in residual evaluation. [2] and [13] have done some systematic studies on threshold computation in deterministic and statistic settings, respectively. Compared with statistic method, the benefits of the deterministic threshold computation methods lie in the simplicity and easy implementation. Moreover, as pointed out in [2], the wellestablished robust control theory provides a systematic way to threshold computation the deterministic framework. Using $H_{\infty}$ and peak-to-peak optimization techniques, Ding et al. propose norm-based threshold computation methods for observer-based fault detection in linear systems [2] and

This work is supported by National Natural Science Foundation of China (Grant No. 61773145), State Key Laboratory of Robotics (Grant No. 2014-O04), and the Fundamental Research Funds for the Central Universities (HIT.KLOF.2015.076).
Lipschitz nonlinear systems [12]. However, the studies in [2] and [12] still have some limitations. First, the system dynamics of the diagnosed system are used in the methods proposed [2] and [12], consequently, the obtained design requires the stability of the open-loop system. Second, the methods in [2] and [12] do not consider the initial error and only produce a constant threshold.

In recent years, set-membership estimation has received much attention and has been used in fault detection $[6,14,15]$. For example, [6] proposes the concept of $H_{-} / L_{\infty}$ fault detection for continuous-time linear parameter-varying systems and then [14] generates this idea to discrete-time Takagi-Sugeno fuzzy systems. In [15], a zonotopic fault detection observer is proposed by integrate $H_{-}$design with zonotope-based set-membership estimation. In these references, residual evaluation is considered in the design of residual generator such that the proposed method can provide a dynamic threshold for decision making in fault detection. In fact, the major merit of set-membership estimation is that it provides an effective and systematic solution to threshold computation in fault detection. However, to the best of our knowledge, threshold computation methods using set-membership estimation have not yet been fully studied. Moreover, a comprehensive study on threshold computation based on different setmembership estimation methods is also needed. In view of this, this paper proposes two threshold computation methods for observer-based fault detection in discrete-time linear systems and compare their performance by numerical simulations.

\section{Problem Formulation}

Consider the following discrete-time linear system

$$
\left\{\begin{array}{l}
x_{k+1}=A x_{k}+B u_{k}+E_{1} f_{k}+D_{1} d_{k} \\
y_{k}=C x_{k}+D_{2} d_{k}+E_{2} f_{k}
\end{array}\right.
$$


where $x_{k} \in \mathbb{R}^{n_{x}}$ is the state, $u_{k} \in \mathbb{R}^{n_{u}}$ is the control input, $y_{k} \in \mathbb{R}^{n_{y}}$ is the measured output, $f_{k} \in \mathbb{R}^{n_{f}}$ is the fault signal, and $d_{k} \in \mathbb{R}^{n_{d}}$ is the unknown input. $A, B$, $C, D_{1}, D_{2}, E_{1}$ and $E_{2}$ are known constant matrices with appropriate dimensions.

Remark 1. Note that the unknown input $d_{k}$ in (1) may contain both process disturbance and measurement noise. For instance, if the considered system has the following form

$$
\left\{\begin{array}{l}
x_{k+1}=A x_{k}+B u_{k}+E_{1} f_{k}+D_{w} w_{k} \\
y_{k}=C x_{k}+D_{v} v_{k}+E_{2} f_{k}
\end{array}\right.
$$

it can be rewritten as (1) by letting

$$
d_{k}=\left[\begin{array}{l}
w_{k} \\
v_{k}
\end{array}\right], D_{1}=\left[\begin{array}{ll}
D_{w} & 0
\end{array}\right], D_{2}=\left[\begin{array}{ll}
0 & D_{v}
\end{array}\right] .
$$

Without loss of generality, we assume that $x_{0}$ and $d_{k}$ are unknown but bounded.

In this paper, two assumptions on the form of uncertainties are used, depending on the adopted threshold computation methods. A natural way to describe uncertainties is using intervals. Therefore, in the zonotope-based method, interval-based representation is used to describe $x_{0}$ and $d_{k}$ as follows

$$
\underline{x}_{0} \leq x_{0} \leq \bar{x}_{0}, \underline{d} \leq d_{k} \leq \bar{d}
$$

where $\underline{x}_{0}$ and $\bar{x}_{0}$ are the lower and upper boundaries of $x_{0}$, respectively, and $\underline{d}$ and $\bar{d}$ are the lower and upper boundaries of $d_{k}$, respectively. However, the interval representation in (2) cannot be directly used in the method based on the $L_{\infty}$ analysis. Therefore, in the second method, we use norm-based uncertainty representation, e.g.

$$
\left\|x_{0}\right\| \leq\left\|x_{0}\right\|_{\infty},\left\|d_{k}\right\|_{2} \leq\|d\|_{\infty}
$$

Note that the interval description can be over-approximated by a norm-based representation at the cost of certain conservatism. This will be shown latter in the simulation part. In this paper, we study threshold computation in observerbased fault detection. For the diagnosed system in (1), we use the following observer-based residual generator

$$
\left\{\begin{array}{l}
\hat{x}_{k+1}=A \hat{x}_{k}+B u_{k}+L\left(y_{k}-C \hat{x}_{k}\right) \\
r_{k}=y_{k}-C \hat{x}_{k}
\end{array}\right.
$$

where $\hat{x}_{k} \in \mathbb{R}^{n_{x}}$ is the state estimation, $r_{k} \in \mathbb{R}^{n_{y}}$ is the residual for fault detection and $L \in \mathbb{R}^{n_{x} \times n_{y}}$ is the gain matrix of the fault detection observer. Note that there have been abundant of results on fault detection observer design in the literature $[6,16,17]$. For instance, we can use $H_{-} / H_{\infty}$ design $[6,16]$ or $H_{-} / L_{\infty}$ method [14] to design the observer in (4). Since the focus of this paper is threshold computation, we assume that matrix $L$ has already been determined.

In the residual evaluation step, it is necessary to calculate the threshold of the residual $r_{k}$ in fault-free case. To this end, we need to analyse the dynamic of $r_{k}$. First, we define the estimation error as

$$
e_{k}=x_{k}-\hat{x}_{k}
$$

In order to calculate the threshold of $r_{k}$, we can first analyse the range of $e_{k}$. By using (1) and (4), the error dynamic can be obtained as

$$
\begin{aligned}
e_{k+1}= & A x_{k}+B u_{k}+D_{1} d_{k}+E_{1} f_{k}-\left(A \hat{x}_{k}+B u_{k}\right. \\
& \left.+L\left(y_{k}-C \hat{x}_{k}\right)\right) \\
= & (A-L C) e_{k}+D_{1} d_{k}-L D_{2} d_{k}+E_{1} f_{k}-L E_{2} f_{k}
\end{aligned}
$$

In addition, using the definition of residual in (4) yields

$$
\begin{aligned}
r_{k} & =y_{k}-C \hat{x}_{k} \\
& =C x_{k}+D_{2} d_{k}+E_{2} f_{k}-C \hat{x}_{k} \\
& =C e_{k}+D_{2} d_{k}+E_{2} f_{k}
\end{aligned}
$$

In threshold computation, we only consider the fault-free case, i.e.

$$
\left\{\begin{array}{l}
e_{k+1}=(A-L C) e_{k}+D_{1} d_{k}-L D_{2} d_{k} \\
r_{k}=C e_{k}+D_{2} d_{k}
\end{array}\right.
$$

In this paper, we will introduce dynamic threshold computation methods based on zonotope and $L_{\infty}$ for (8) system. Then, the performances of the two methods will be compared through several simulation studies. The main objective of this paper is to compare the two methods to choose a more accurate one.

\section{Two Threshold Computation Methods}

\subsection{Threshold computation based on zonotopes}

In this subsection, the system variables and uncertain parameters are described by the totally symmetric polytope: zonotope. The definition of zonotope is as follows Definition 1 - A m-order zonotope in $\mathbb{R}^{n}$ is the translation by the center $p \in \mathbb{R}^{n}$ of the linear image of an unitary hypercube of dimension $m$. Given a matrix $H \in \mathbb{R}^{n \times m}$ representing the linear transformation, the zonotope $\mathcal{Z}$ is defined by

$$
\mathcal{Z}=\langle p, H\rangle=\left\{p+H z: z \in \mathbb{B}^{n}\right\}
$$

where $p \in \mathbb{R}^{n}$ is the center of $\mathcal{Z}, H \in \mathbb{R}^{n \times m}$ is called the generator matrix of $\mathcal{Z}$ which determines the shape and volume. In order to simplify the symbol, we use $\langle p, H\rangle$ to describe a zonotope $\mathcal{Z}$. So the initial value of the state error, the process disturbance and the measurement noise satisfy

$$
\left\{\begin{array}{l}
e_{0} \in\left\langle p_{0}, H_{0}\right\rangle \\
d_{k} \in\left\langle 0, H_{d}\right\rangle
\end{array}\right.
$$

For the following proof, the following properties of zonotopes are introduced

The Minkowski sum of two zonotope is also a zonotope

$$
\left\langle p_{1}, H_{1}\right\rangle \oplus\left\langle p_{2}, H_{2}\right\rangle=\left\langle p_{1}+p_{2},\left[\begin{array}{ll}
H_{1} & H_{2}
\end{array}\right]\right\rangle
$$

The linear image of a zonotope by a matrix $L$ satisfies

$$
L\langle p, H\rangle=\langle L p, L H\rangle
$$

A zonotope can be enclosed by another one

$$
\langle p, H\rangle \subseteq\langle p, \hat{H}\rangle
$$


where $p, p_{1}, p_{2} \in \mathbb{R}^{n}, H, H_{1}, H_{2} \in \mathbb{R}^{n \times m}, L \in \mathbb{R}^{l \times n}$ is matrices of appropriate dimensions. $\hat{H} \in \mathbb{R}^{n \times n}$ is a diagonal matrix, the elements is

$$
\hat{H}_{i i}=\sum_{j=1}^{m}\left|H_{i j}\right|, i=1, \cdots, n
$$

Note that the dimensions of the zonotopes are increasing with time, the amount of computation will be eventually beyond the computing power of the computer. Therefore, dimension reduction operator should be used to reduce the computation burden of zonotopes.

Lemma 1. Consider an m-order zonotope $\mathcal{Z}=\langle p, H\rangle$, $H \in \mathbb{R}^{n \times m}$ and integer $s$, where $n \leq s \leq m, \hat{H}$ is a matrix whose columns are those of $H$ reordered in decreasing Euclidean norm, then the dimension of zonotope can be reduced from $m$ to $s$

$$
\mathcal{Z} \in\left\langle p,\left[\begin{array}{ll}
\hat{H}_{1} & Q
\end{array}\right]\right\rangle
$$

where $\hat{H}_{1}$ is the first s-n columns of $\hat{H}, \hat{H}_{2}$ is the rested part of $\hat{H}, Q$ is a diagonal matrix which can be obtained by the following eqution according to (14)

$$
Q_{i i}=\sum_{j=1}^{m}\left|\hat{H}_{2}\right|_{i j}, i=1, \cdots, n
$$

The following theorem are proposed to calculate the threshold for the residual by zonotope-based method.

Theorem 1. Assuming $e_{k} \in\left\langle p_{k}, \hat{H}_{k}\right\rangle$, then according to (8) and (10), $e_{k+1}$ satisfies

$$
e_{k+1} \in\left\langle p_{k+1}, H_{k+1}\right\rangle
$$

where

$$
\left\{\begin{array}{l}
p_{k+1}=(A-L C) p_{k} \\
H_{k+1}=\left[\begin{array}{ll}
(A-L C) H_{k} & \left(D_{1}-L D_{2}\right) H_{d}
\end{array}\right]
\end{array}\right.
$$

Proof. The error dynamic equation can be obtained:

$$
e_{k+1}=(A-L C) e_{k}+\left(D_{1}-L D_{2}\right) d_{k}
$$

By the linear property of zonotope, we have

$$
\left\{\begin{array}{l}
(A-L C) e_{k} \in\left\langle(A-L C) p_{k},(A-L C) H_{k}\right\rangle \\
\left(D_{1}-L D_{2}\right) d_{k} \in\left\langle 0,\left(D_{1}-L D_{2}\right) H_{d}\right\rangle
\end{array}\right.
$$

By using (19) and the property of zonotope, we have

$$
e_{k+1} \in\left\langle p_{k+1}, H_{k+1}\right\rangle
$$

where

$$
\left\{\begin{array}{l}
p_{k+1}=(A-L C) p_{k} \\
H_{k+1}=\left[\begin{array}{ll}
(A-L C) H_{k} & \left(D_{1}-L D_{2}\right) H_{d}
\end{array}\right]
\end{array}\right.
$$

After obtaining the zonotope representation of any time of $e_{k} \in\left\langle p_{e_{k}}, H_{e k}\right\rangle$, we can get the bound of residual $r_{k}$ to be the threshold

$$
r_{k} \in\left\langle C p_{e_{k}}, H_{r_{k}}\right\rangle
$$

where

$$
H_{r_{k}}=\left[\begin{array}{ll}
C H_{e_{k}} & D_{2} H_{d}
\end{array}\right]
$$

\subsection{Threshold computation based on $L_{\infty}$ analysis}

For the system (1), supposing that a stable residual generator (4) has been designed, the state estimation error vector $e_{k}$ and the generated residual $r_{k}$ have been obtained as

$$
\left\{\begin{array}{l}
e_{k+1}=(A-L C) e_{k}+E_{1} f_{k}+\left(D_{1}-L D_{2}\right) d_{k} \\
r_{k}=C e_{k}+E_{2} f_{k}+D_{2} d_{k}
\end{array}\right.
$$

where the initial conditions of the system and the disturbance satisfy

$$
\underline{e}_{o} \leq e_{0} \leq \bar{e}_{0},\left\|d_{k}\right\|_{2} \leq\|d\|_{\infty}
$$

The following theorem is proposed to design the $L_{\infty}$ threshold of residual.

Theorem 2. Given positive scalar $\alpha$ and $\gamma$, if there exists a symmetric positive definite matrix $P=P^{T} \in \mathbb{R}^{n_{x} \times n_{x}}$, and the following conditions hold

$$
\begin{gathered}
{\left[\begin{array}{cc}
S_{1} & S_{2} \\
S_{2}^{T} & S_{3}
\end{array}\right] \prec 0} \\
\|C\|_{2}^{2}-\gamma^{2} P \prec 0
\end{gathered}
$$

where

$$
\left\{\begin{array}{l}
S_{1}=(A-L C)^{T} P(A-L C)-\alpha P \\
S_{2}=(A-L C)^{T} P\left(D_{1}-L D_{2}\right) \\
S_{3}=\left(D_{1}-L D_{2}\right)^{T} P\left(D_{1}-L D_{2}\right)-(1-\alpha) I
\end{array}\right.
$$

then the residual sastifies

$$
\left\|r_{k}\right\|_{2} \leq \sqrt{\gamma^{2} \alpha^{k} \bar{V}(0)+\left(\gamma^{2}+\left\|D_{2}\right\|_{2}^{2}\right)\left\|d_{k}\right\|_{\infty}^{2}}
$$

where $\bar{V}(0)$ is the upper bound of the initial value of the selected Lyapunov function

$$
V(0)=e_{0}^{T} P e_{0} \leq \lambda_{\max }(P)\left\|e_{0}\right\|_{\infty}^{2}
$$

Proof. Setting $f_{k}=0$, the state estimation error system which only effected by disturbance and noise is written as

$$
\left\{\begin{array}{l}
x_{k+1}=A x_{k}+B u_{k}+D_{1} d_{k} \\
y_{k}=C x_{k}+D_{2} d_{k}
\end{array}\right.
$$

A Lyapunov function is chosen as

$$
V(k)=e_{k}^{T} P e_{k}
$$

Given a positive scalar $0<\alpha<1$, under zero initial conditions, if the following inequality holds

$$
V(k+1)<\alpha V(k)+(1-\alpha) d_{k}^{T} d_{k}
$$

then, we have

$$
\begin{aligned}
V(k) & <\alpha^{k} V(0)+(1-\alpha) \sum_{i=0}^{k-1} \alpha^{i} d_{k}^{T} d_{k} \\
& <\alpha^{k} V(0)+(1-\alpha) \sum_{i=0}^{k-1} \alpha^{i}\|d\|_{\infty}^{2} \\
& <\alpha^{k} V(0)+\left(1-\alpha^{k-1}\right)\|d\|_{\infty}^{2} \\
& <\alpha^{k} V(0)+\|d\|_{\infty}^{2}
\end{aligned}
$$


Furthermore, if it is satisfied in (27), such that

$$
\|C\|_{2}^{2}<\gamma^{2} P
$$

then, it obtains

$$
\left\|C e_{k}\right\|_{2}^{2}<\gamma^{2} e_{k}^{T} P e_{k}
$$

According to the definition of the residual $r_{k}$ in (24), we have

$$
\left\|r_{k}\right\|_{2}^{2} \leq\left\|C e_{k}\right\|_{2}^{2}+\left\|D_{2}\right\|_{2}^{2}\|d\|_{2}^{2}
$$

Based on (34) and (36), and the priori condition and initial state $(25)$ we obtain that

$$
\begin{aligned}
\left\|r_{k}\right\|_{2} & \leq \sqrt{\gamma^{2} P\left\|e_{k}\right\|_{2}^{2}+\left\|D_{2}\right\|_{2}^{2}\|d\|_{\infty}^{2}} \\
& \leq \sqrt{\gamma^{2} \alpha^{k} V(0)+\left(\gamma^{2}+\left\|D_{2}\right\|_{2}^{2}\right)\|d\|_{\infty}^{2}} \\
& \leq \sqrt{\gamma^{2} \alpha^{k} \bar{V}(0)+\left(\gamma^{2}+\left\|D_{2}\right\|_{2}^{2}\right)\|d\|_{\infty}^{2}}
\end{aligned}
$$

Substituting (32) into (33), we have

$$
\left[\begin{array}{c}
e_{k} \\
d_{k}
\end{array}\right]^{T}\left[\begin{array}{ll}
S_{1} & S_{2} \\
S_{2}^{T} & S_{3}
\end{array}\right]\left[\begin{array}{c}
e_{k} \\
d_{k}
\end{array}\right]<0
$$

then (26) can be derived from (39).

In order to get a more accurate threshold, we need to get a smallest $\gamma$ when the $\alpha$ is given, the observer gain matrix is transformed by solving the following optimization problem

$$
\min _{\text {s.t. }(26)-(27)} \gamma
$$

As $\alpha$ is an artificial selection scalar, it needs to analyze the relationship between $\alpha$ and $\gamma$ to make the $\gamma$ lowest. In this paper, the scalar $\alpha$ is selected by linear searching method.

\section{Simulations}

In this section, we use the linearized dynamic model of a vertical take-off and landing aircraft borrowed from [17] to test and compare the performance of the aforementioned two threshold computation methods. The considered system has the form of (1) with

$$
\begin{gathered}
A=\left[\begin{array}{cccc}
0.9828 & 0.0083 & -0.0454 & -0.2461 \\
0.0117 & 0.5813 & -0.3898 & -1.6662 \\
0.0458 & 0.1274 & 0.8230 & 0.4803 \\
0.0117 & 0.0358 & 0.4433 & 1.1361
\end{array}\right], \\
B_{1}=\left[\begin{array}{cc}
0.2666 & 0.0365 \\
1.7629 & -3.2664 \\
-2.3152 & 1.7209 \\
-0.6083 & 0.4660
\end{array}\right], D_{1}=\left[\begin{array}{cc}
-0.0069 & 0.0026 \\
0.0688 & 0.3896 \\
0.1144 & 0.0358
\end{array}\right],
\end{gathered}
$$

$E_{1}=B_{1}, C=\left[\begin{array}{llll}1 & 0 & 0 & 0 \\ 0 & 1 & 0 & 0 \\ 0 & 0 & 1 & 0 \\ 0 & 1 & 1 & 1\end{array}\right], D_{2}=\left[\begin{array}{cc}0 & 0.2 \\ 0 & 0.1 \\ 0.3 & 0 \\ 0 & 0\end{array}\right], E_{2}=0$.

In the simulation, we use the fault detection observer in (4) with the following gain matrix

$$
L=\left[\begin{array}{cccc}
-0.1400 & 0.3210 & 0.1638 & -0.4014 \\
0.8187 & 1.1276 & -0.1521 & -0.2019 \\
0.6713 & -0.1058 & 1.1504 & 0.1467 \\
0.3658 & -0.5171 & 0.1117 & 0.4864
\end{array}\right]
$$

Note that the considered threshold computation methods use different geometric sets to describe $d_{k}$. Although the interval-based uncertainty set can be over-approximated by a norm-based representation, and vice versa, the overapproximation will bring certain conservatism. Therefore, in order to fairly compare the performance of these two methods, two cases with different form of $d_{k}$ are conducted in the simulation.

Case 1 . In the first case, $d_{k}$ is randomly generated from the following set

$$
\left[\begin{array}{l}
-0.5 \\
-0.5
\end{array}\right] \leq d_{k} \leq\left[\begin{array}{l}
0.5 \\
0.5
\end{array}\right]
$$

This is an interval-based set, hence it is suitable for zonotope-based threshold computation. However, to use the $L_{\infty}$ threshold computation method, one should construct a norm-based set to over-approximate this intervalbased set. In this case, the sets used in these two threshold computation methods are depicted in the Fig. 1, where the interval-based uncertainty set is in red and the norm-based uncertainty set is in blue.

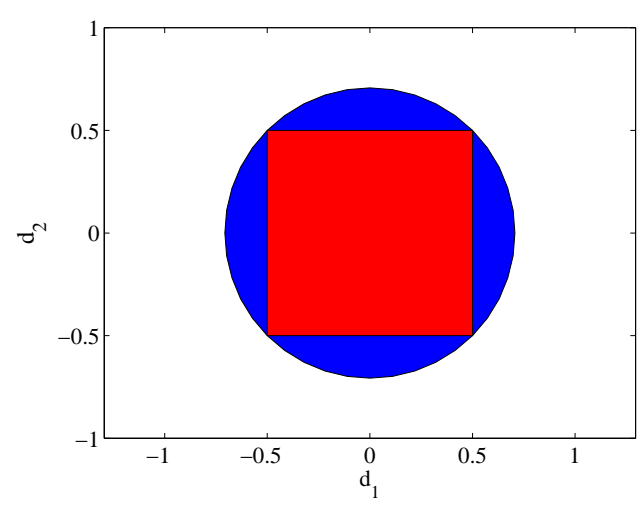

Figure 1: The uncertainty sets in Case 1.

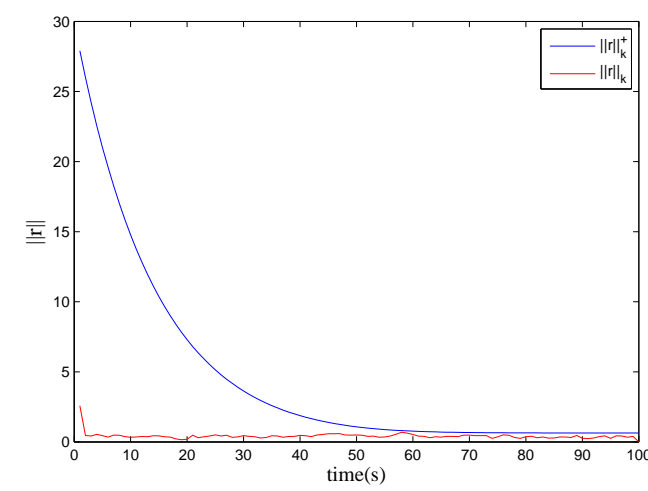

Figure 2: The maximum fault that cannot be detected by the $L_{\infty}$-based method in Case 1 .

To compare the two methods, we consider the following fault as a unit fault function

$$
f_{0}=\left\{\begin{array}{ll}
{\left[\begin{array}{ll}
1 & 0
\end{array}\right]^{T},} & \text { if } k \geq 20 \\
{\left[\begin{array}{ll}
0 & 0
\end{array}\right]^{T},} & \text { if } k<20
\end{array} .\right.
$$


Through simulations, we found that the maximum fault that cannot be detected by the $L_{\infty}$-based method is $0.14 f_{0}$. The fault detection result is depicted in Fig. 2. In this situation, if we apply the zonotope-based method, we will get the fault detection result in Fig. 3. From Fig. 3, it can be seen that $r_{3}$ and $r_{4}$ occasionally exceed the threshold. This implies that the performance of the zonotope-based method is a little better than the $L_{\infty}$ method.
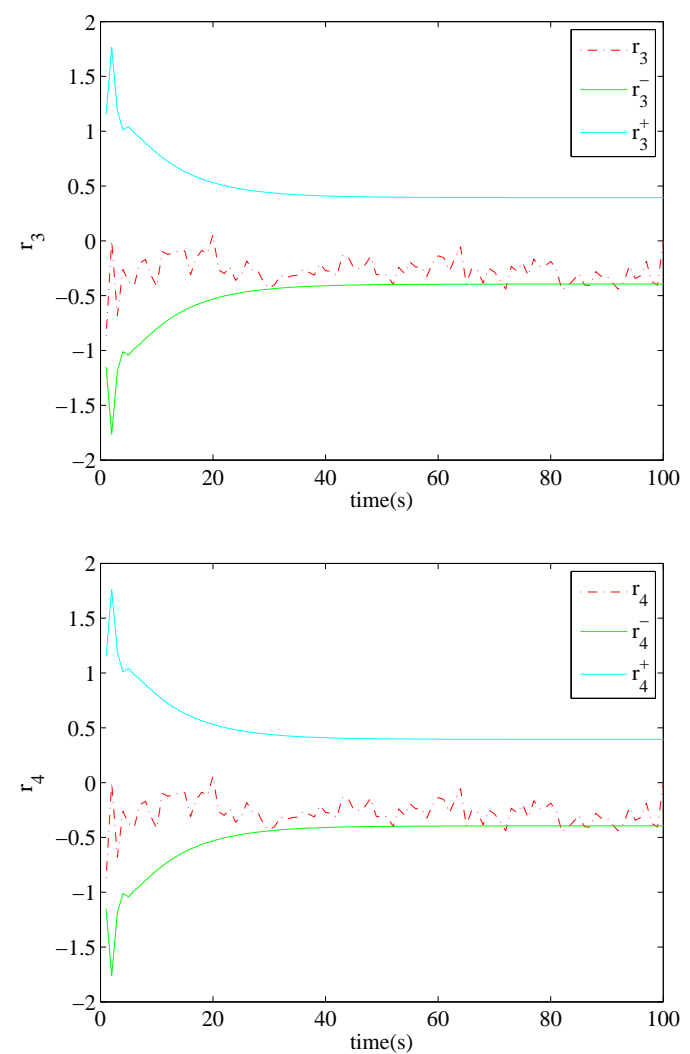

Figure 3: The fault detection result by the zonotope-based method in Case 1.

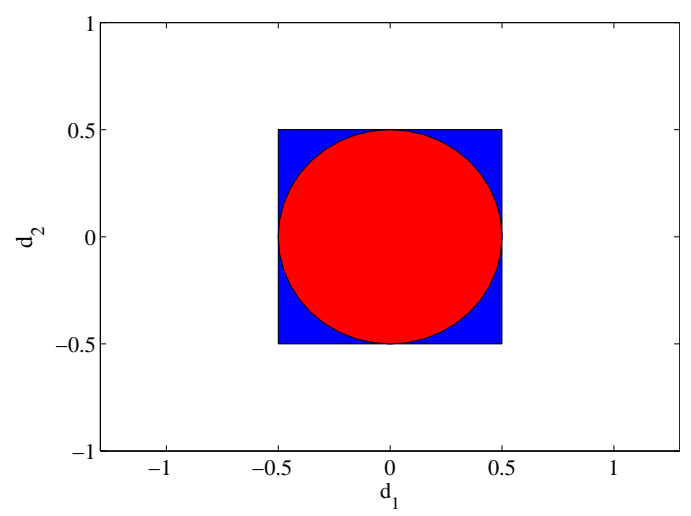

Figure 4: The uncertainty sets used by the two threshold computation methods in Case 2.

Case 2. In the second case, the considered $d_{k}$ has the following form

$$
d_{k}=\left[\begin{array}{l}
0.5 \sin (k) \\
0.5 \cos (k)
\end{array}\right]
$$

In this case, the uncertainty sets in the studied threshold computation methods are shown in Fig. 4, where the interval-based uncertainty set is in red and the norm-based uncertainty set is in blue. It is obvious that the intervalbased uncertainty set has certain conservatism compared to the norm-based one.

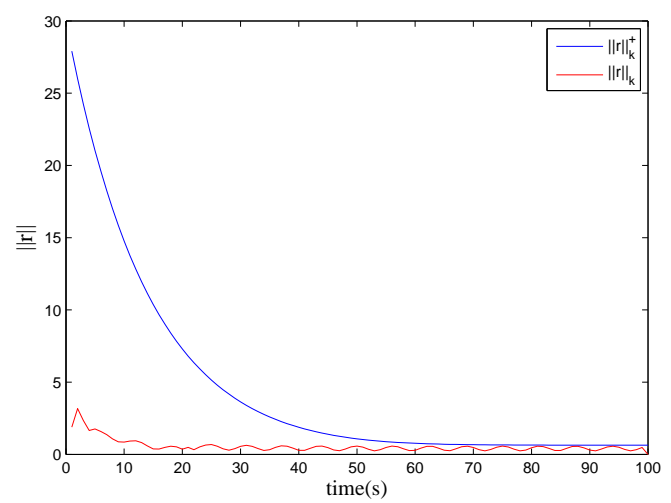

Figure 5: The maximum fault that cannot be detected by $L_{\infty}$-based method in Case 2.
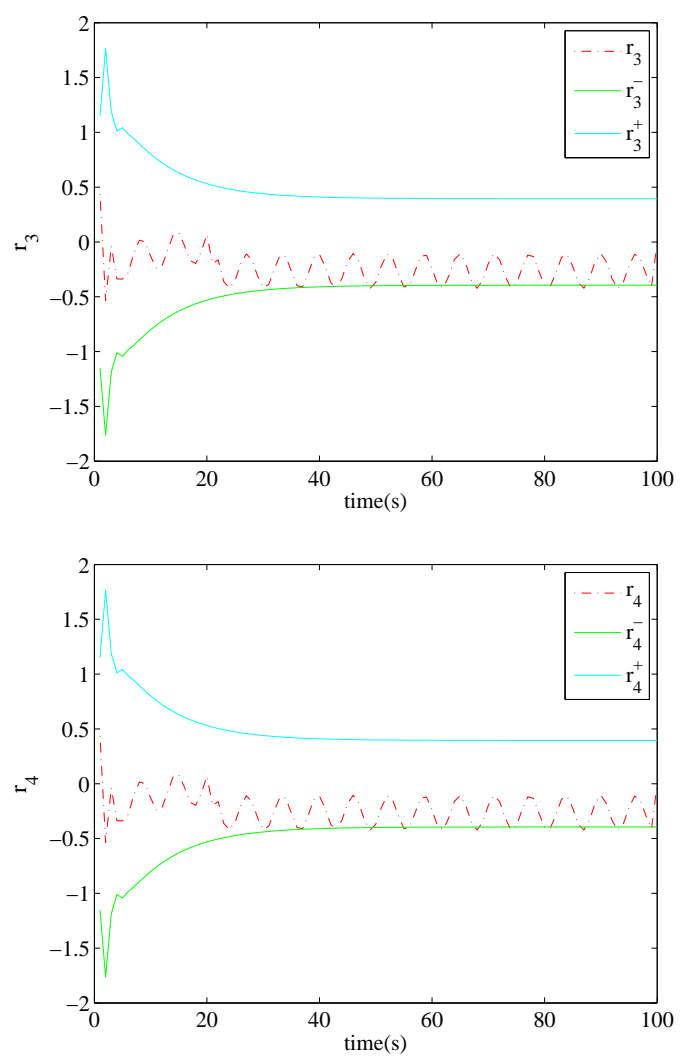

Figure 6: The fault detection result by the zonotope-based method in Case 2.

By simulations, we found that the maximum fault that cannot be detected by the $L_{\infty}$-based method as $0.13 f_{0}$, as shown in Fig. 5. By applying the zonotope-based method, we can get the result in Fig. 6. In this situation, the zonotope-based method can detect the fault, but it also almost reaches the detection limit. 


\section{Conclusion}

In this paper, we propose two threshold computation methods for observer-based fault detection of discrete-time linear systems. The first method assumes that the uncertainties are bounded in known intervals and the second one assumes the uncertainties to be norm-bounded. The main contribution of this paper is that it proposes systematic methods for residual evaluation based on reasonable assumptions that the uncertainties are unknown but bounded. Numerical simulations are given to verify and compare the performance of the proposed methods. It is noted that the residual evaluation problem is still challenging and future works need to be done to further reduce the conservatisms of the threshold computation methods.

\section{REFERENCES}

[1] J. Chen and R. Patton, Robust Model-Based Fault Diagnosis for Dynamic Systems, New York: Springer, 1999.

[2] S. X. Ding, Model-Based Fault Diagnosis Techniques: Design Schemes, Algorithms and Tools, Berlin: Springer, 2008.

[3] K. Zhang, B. Jiang and P. Shi, Observer-Based Fault Estimation and Accommodation for Dynamic Systems, Berlin: Springer, 2013.

[4] M. Hou and R. Patton, An LMI approach to $H_{-} / H_{\infty}$ fault detection observer, Proceedings of the UKACC International Conference on Control, 305-310, 1996.

[5] M. Zhou, Z. Wang, Y. Shen and M. Shen, $H_{-} / H_{\infty}$ fault detection observer design in finite-frequency domain for Lipschitz nonlinear systems, IET Control Theory \& Applications, Vol.11, No.14, 2361-2369, 2017.

[6] Z. Wang, S. Peng and C.C. Lim, $H_{-} / H_{\infty}$ fault detection observer in finite frequency domain for linear parametervarying descriptor systems, Automatica, Vol.86, 38-45, 2017.

[7] J. Gertler and R. Monajemy, Generating directional residuals with dynamic parity relations, Automatica, Vol.31, No.4, 627-635, 1995.

[8] M. Zhong, S. X. Ding, Q. Han and Q. Ding, Parity spacebased fault estimation for linear discrete time-varying systems, IEEE Trans. on Automatic Control, Vol.55, No.7, 1726-1731, 2010.

[9] A. Emami-Naeini, M. M. Akhter and S. M. Rock, Effect of model uncertainty on failure detection: the threshold selector, IEEE Trans. on Automatic Control, Vol.33, No.12, 1106$1115,1988$.

[10] P. M. Frank and S. X. Ding, Frequency domain approach to optimally robust residual generation and evaluation for model-based fault diagnosis, Automatica, Vol.30, No.5, 789804, 1994.

[11] T. Hsiao and M. Tomizuka, Threshold selection for timely fault detection in feedback control systems, American Control Conference, 3303-3308, 2005.

[12] A. Q. Khan and S. X. Ding, Threshold computation for fault detection in a class of discrete-time nonlinear systems, International Journal of Adaptive Control Signal Processing, Vol.25, No.5, 407-429, 2011.

[13] M. Basseville and I. Nikiforov, Detection of abrupt changes: theory and application, Englewood Cliffs: Prentice Hall, 1993.

[14] M. Zhou, M. Rodrigues, Z. Wang, Y. Shen, and D. Theilliol, $H_{-} / L_{\infty}$ fault detection observer for discrete-time TakagiSugeno fuzzy systems, IEEE Conference on Decision and Control, accepted, 2017.
[15] Y. Wang, M. Zhou, V. Puig, G. Cembrano, and Z. Wang, Zonotopic fault detection observer with $H_{-}$performance, Chinese Control Conference, 7230-7235, 2017.

[16] M. Chadli, A. Abdo, and S. X. Ding, $H_{-} / H_{\infty}$ Fault detection filter design for discrete-time Takagi-Sugeno fuzzy system, Automatica, Vol.49, No.7, 1996-2005, 2013.

[17] H. Wang and G. Yang, A finite frequency domain approach to fault detection observer design for linear continuous-time systems, Asian Journal of Control, Vol.10, No.5, 559-568, 2008. 